\title{
I. Ueber die Verwachsung von Allanit (Orthit) und Epidot in Gesteinen.
}

\author{
Von W. H. Hobbs aus Worcester, Mass., U. S. A.
}

(Mit 2 Textfiguren.)

Das Zusammenauftreten von Allanit und Epidot in Gesteinen ist nicht ganz unbekannt. Schon im Jahre 1854 erwähnte Blomstrand ${ }^{1}$ ) von Wexiö in Schweden Pistazitkrystalle radialstrahlig um Allanitkerne geordnet und später Ewald Becker²) Allaniteinschliisse in Orthoklas und Epidot. Cross und Idding $\mathrm{s}^{3}$ ) machten aufmerksam auf scheinbare Epidoteinschlüsse in Allanit. In vielen Lehrbüchern findet man angegeben, dass bei Sillböle in Finnland Kerne von Allanit in Epidot und Kerne von Epidot in Allanit vorkommen. Der Liebenswiirdigkeit des Prof. F. J. Wiik von Helsingfors verdanke ich einen Abzug des Katalogs der Mineralsammlung zu Helsingfors ${ }^{4}$, in dem ein Epidotkrystall mit Allanitkern von Sillböle abgebildet ist. 1882 beschrieb Törnebohmb) im Epidotgneiss

1) B l o m stra nd, Oefvers. af akad. Förband. Jahrgang 1854, Nr. 9, pag. 296. - Ref. Journ. f. prakt. Chemie. LXVI, pag. 156.

2) Ewald Becker, Ueber das Mineralvorkommen im Granit von Striegau, insbesondere über den Orthoklas und dunkelgrünen Epidot. Breslau.

s) Cross und Iddings, Widespread occurrence of Allanit as an accessory constituent of many rocks. Am. Journ. Sci. [3] Aug. 1885, XXXII, pag. 108,

4) F. J. Wiik, Den Finska Mineralsamlingen i universitets i Helsingfurs Mineralkabinett. 1887 , pag. 27 a. Taf. II.

5) A. E. Törnebohm, Mikroskopiska bergartsstndier. XII. Epidotgneiss. Geol. För. i Stockholm Förhandl. 1882, Nr. 75, Bd. VI, Heft 5, pag. 189.

Nineralog. und petrogr. Mitth. 1889. XI. (W. H. Hobbs. R. Prendel.) 
Wermlands eine Verwachsung von Epidot mit einem für Orthit gehaltenen Minerale. "Als Einschliusse kommen dem Epidot krystallographisch ähnliche und mit dem Wirth gleich orientirte Kryställehen vor, welche für Orthit gehalten werden. " 1) Zwillingsbildungen waren den beiden Mineralen gemeinsam. Der Orthit wandelt sich in braungelbes Pulver um.

Als ich im letzten Jahre ein Gebiet in der Umgebung von Baltimore einer Untersuchung unterwarf, ïberraschte mich die bedeutsame Rolle, die die Minerale Epidot und Allanit im Granit von Ilchester spielen. Eine vorläufige Notiz ${ }^{2}$ ) über die geologischen Verhältnisse dieser Gegend ist im April 1888 erschienen.

Der Granit von Ilchester ist eines der jüngsten in einer Reihe von intrusiven Gesteinen, welche in den krystallinischen Schiefern Marylands auftreten. Es ist ein mittel- bis grobkörniges Gestein mit einem durch grosse Mikroklinkrystalle bedingten porphyrartigen Charakter und seinem Aussehen nach dem Amphibolgranit ${ }^{3}$ ) vom Birkenauerthale im Odenwald älnlich. Die holokrystalline, auch wohl granophyrische Grundmasse besteht aus wechselnden Mengen von ortho- und klinotomem Feldspathe. Das Hauptgestein enthält als wesentlichen Gemengtheil auch Glimmer, und zwar zum grossen Theile Biotit mit einer untergeordneten Menge von hellem Glimmer. Es gibt auch Facies, wo Hornblende an die Stelle des Glimmers tritt. Durch das ganze Gebiet sieht man Druckerscheinungen, insbesondere eine mehr orter weniger deutliche Parallelstructur.

Schon mit blossem Ange erkennt man im Gestein gelbgefïrbte, ein bis drei Millimeter lange säulenfömige Krystalle ron epidotähnlichem Aussehen, wcleho sich sehr gern nesterweise zusammenhäufen und unregelmässig verwachsen. Unmittelbar im Centrum eines solchen Epidotkrystalls und von demselben seharf begrenzt, sieht man nicht selten einen tiefbraunen Kern, der mit der Loupe sich als ein mit dem gelben Epidot gleich orientirter und durch dieselhe Ansbildungsform charakterisirter Krystall erkennen lässt. Diese Kerne zeigen keine dentliche Spaltbarkeit und zerfallen sehr

1) Ref. Neues Jahrbuch (Cohen). 1883, Bd. I, pag. 2łう.

2) W. H. Hob bs, On the rocks of Ilchester etc. Johns Hopkins University Circulars Nr. 6ว. April 1888.

$\left.{ }^{3}\right)$ Benecke and Cohen, Geogn. Beschreibnng der Umgebung von Heidelberg. Strassburg 1879-81. 
leicht in ein braunes erdiges Pulver, während der Epidot ganz unverändert bleibt. In den weitaus meisten Fällen sind zwei Flächen einer prismátischen Zone deutlich sichtbar, welche entsprechenden Flächen des Epidots parallel laufen. Diese Beobachtung deutet darauf hin, dass der Allanit parallel dem Epidot eingeschaltet ist. Der Allanit ist sehr spröde und lässt sich nicht leicht herauslösen. Deswegen waren Winkelmessungen nicht möglich.

Unter dem Mikroskope sieht man den Epidot zum Theil idiomorph, zum Theil allotriomorph gegen den Biotit und den. Feld-

Fig. 1.

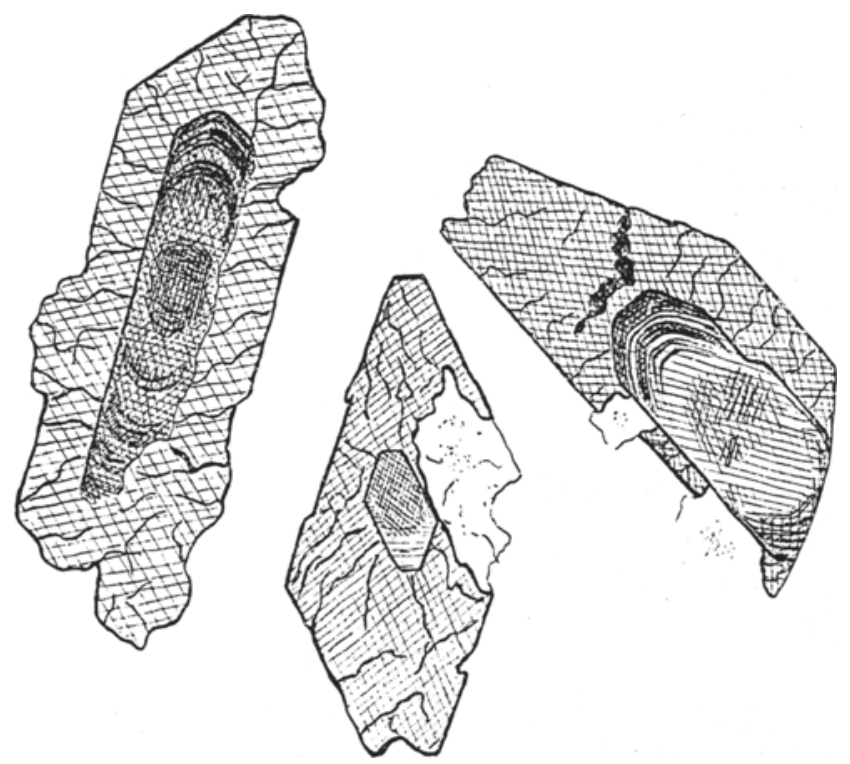

Längs- und Querschnitte der Epidot-Allanit-Verwachsungen im Ilchestergranit. Vergrösserung 50.

spath begrenzt. Die Farbe ist gelb bis hellgriun. Die Längsschnitte löschen gerade aus und besitzen hohe Licht- und Doppelbrechung. Die Interferenzfarben zwischen gekreuzten Nicols erreichen die der dritten oder vierten Ordnung, je nach der Dicke des Schliffes. Solche Längsschnitte lassen eine optische Axe oft am Rande des Gesichtsfeldes austreten und mit Sicherheit ist zu erkenmen, dass die Ebene der optischen Axen senkrecht zur Längsrichtung, also senkrecht zur Orthodiagonale liegt. Der Axenwinkel scheint gross zu sein. Die Dispersion ist schwach mit $p>\%$. Die Querschnitte des Minerals 
sind durch $M(001), r(\overline{101})$ und untergeordnetes $T(100)$ begrenzt und zeigen Spaltbarkeit unvollkommen nach $M$ und weniger deutlich nach $T$. Der Winkel $M: T$ wurde zu $115^{\circ}$ gemessen. Die Axe grösster Elasticität liegt im spitzen Winkel $\beta$, nur wenig schief zur verticalen Axe geneigt. Der Winkel $\mathfrak{a}: c$ beträgt $3^{0}$ bis $3^{0} 3^{3}$. Der Pleochroismus ist deutlich, und zwar: a fast farblos, $\mathfrak{b}$ schwach strohgelb, $\mathfrak{c}$ zeisiggrïn. Die Absorption wird ansgedrïckt durch $\mathfrak{c}>\mathfrak{b}>\mathfrak{a}$. Durch Benutzung der Th o u le t'schen Lösung wurde Epidot zusammen mit etwas Allanit und Titanit von den übrigen Gemengtheilen getrennt. Der Epidot liess sich in reinem Zustande nur durch sorgfältiges Auslesen mit der Lupe erhalten. Das specifische Gewicht einer 0.45 Gramm betragenden, auf diese Weise erhaltenen Menge des Epidots wurde mit dem Pyknometer zu 3.39 bestimmt. Der geologischen Landesanstalt der Vereinigten Staaten rerdanke ich eine Analyse dieses Pulvers von Herrn Dr. W. F. Hillebrand, welcher mit besonderer Sorgfalt auf das Vorhandensein der seltenen Erden prifte, ohne aber eine Spur derselben nachweisen zu können. Die Resultate folgen unter I. Thonerde wurde wegen der kleinen Menge des Analysenmaterials nur aus der Differenz bestimmt. Der Eisengehalt ist vielleicht zum Theil Eisenoxydul. Analyse II ist eine Analyse des Untersulzbacher Epidots von Ludwig. Betrachtet man die kleine Menge $P_{2} O_{5}$ in I als von eingeschlossenem Apatit herrührend und berechnet man den Mangan- und Magnesiagehalt mit dem Kalkgehalt zusammen, so erhält man aus Analyse I die Werte in III. Nimmt man weiter an, dass Epidot aus den zwei Molekiulen $\mathrm{H}_{2} \mathrm{Ca}_{4} \mathrm{Al}_{6} \mathrm{Si}_{6} \mathrm{O}_{26}$ und $\mathrm{H}_{2} \mathrm{Ca}_{4} \mathrm{Fe}_{6} \mathrm{Si}_{6} \mathrm{O}_{26}$ besteht, so ist IV die Zusammensetzung einer Mischung aus $2 \mathrm{H}_{2} \mathrm{Ca}_{4} \mathrm{Al}_{6} \mathrm{Si}_{6} \mathrm{O}_{26}+$ $\mathrm{H}_{2} \mathrm{Ca}_{4} \mathrm{Fe}_{6} \mathrm{Si}_{6} \mathrm{O}_{20}$, welche III nahe steht.

\begin{tabular}{|c|c|c|c|c|c|}
\hline & & I. & II & III & 18 \\
\hline $\mathrm{SiO}_{2}$ & & $37 \cdot 63$ & $37 \cdot 83$ & $37 \cdot 80$ & $37 \cdot 44$ \\
\hline $\mathrm{Al}_{2} \mathrm{O}_{3}$ & & $20 \cdot 86$ & 22.63 & 20.95 & $22 \cdot 61$ \\
\hline $\mathrm{Fe}_{2} \mathrm{O}_{3}$ & & $15 \cdot 29$ & 1502 & $15 \cdot 30^{\circ}$ & $14 \cdot 79$ \\
\hline $\mathrm{FeO}$ & & & $0 \cdot 93$ & 1000 & - \\
\hline$M n O$. & . & 0.31 & - & - & - \\
\hline CaO & & 22.93 & $23 \cdot 27$ & $23 \cdot 65$ & $23 \cdot 29$ \\
\hline $\mathrm{MgO}$. & • & 0.31 & - & - & - \\
\hline $\mathrm{H}_{2} \mathrm{O}$. & . & $2.23 G l . V$ & $V .2 \cdot 05$ & $2 \cdot 24$ & $1 \cdot 87$ \\
\hline$P_{2} O_{5}$ & & 0.44 & - & - & - \\
\hline & & 100.00 & $101 \cdot 73$ & 100.00 & $100 \cdot 00$ \\
\hline
\end{tabular}


Die Längsschnitte des Allanits zeigen gerade Auslöschung und hohe Lichtbrechung, genal wie dies beim Epidot der Fall ist. Sie unterscheiden sich aber von diesem dadurch, dass die Doppelbrechung verhältnismässig niedrig ist, so dass die Interferenzfarben zwischen gekreuzten Nicols selbst bei bedeutender Dicke des Schliffes noch innerhalb der ersten Ordnung bleiben. Sie unterscheiden sich auch in auffallender Weise durch ihre tiefbraune Farbe und ausgezeichnete Zonarvertheilung derselben, welche beim Epidot ganz fehlt. Wenn nicht zu tief gefärbt, liefern solche Schnitte eine optische Axe, welche hohe Dispersion zeigt und erkennen lässt, dass die Axenebene senk-

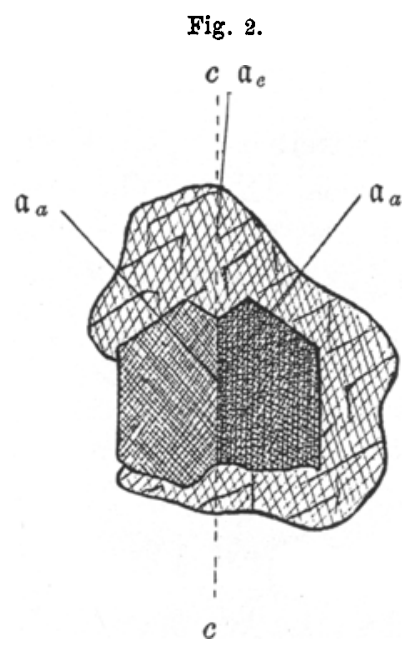

Querschnitt eines nach $T(100)$ verzwillingten Allanitkrystalls mit Epidotumhüllung.

recht zur Längsrichtung liegt. Der Axenwinkel ist wahrscheinlich gross. In Querscbnitten ist eine schlechte Spaltbarkeit nach $r$ wahrnehmbar und selten lässt sich Zwillingsbildung nach der Fläche $T$ beobachten. Ein gliucklicher zufälliger Querschnitt eines solchen Zwillings ist in Fig. 2 abgebildet. In diesem Schnitte, welcher ungefähr senkrecht zur Orthodiagonale liegt, hat der Allanitzwilling gleich grosse Auslöschung auf beiden Seiten der Zwillingsnaht. Die Axe der grössten Elasticität macht mit der Zwillingsnaht, also mit der verticalen Axe, einen Winkel von $36^{\circ}$. Der umbillende Epidot ist ein einheitliches Individuum mit einer Auslöschungsrichtung, welche wenig schief zur Zwillingsnaht läuft. Der Winkel $\mathfrak{a}: c$ beträgt $3^{0}$. 
Der Pleochroismus des Allanits ist stark, und zwar ist $\mathfrak{a}$ schwach gelbbraun, $\mathfrak{b}$ kastanienbraun and $\mathcal{c}$ dunkel grünlichbraun. Die $\mathrm{Ab}-$ sorption ist $\mathfrak{c}>\mathfrak{b}>\mathfrak{a}$. Durch die Liebenswürdigkeit des Herrn Dr. Whitman Cross war ich in der Lage, die Dünnschliffe des Allanits vom Jen Mile Region in Colorado als Vergleichsmaterial benutzen zu können. Der Vergleich zeigt, dass die einzigen wahrnehmbaren Unterschiede in der tieferen Färbung des Coloradominerals und in der Paragenesis des Ilchester Allanits mit Epidot liegen. Diese Verwachsungen von Epidot und Allanit sind in dem Granitmassiv von Ilchester allenthalben verbreitet. ${ }^{1)}$ Selten umschliesst der Allanit einzelne kleine Nadeln, die mit Wahrscheinlichkeit dem Apatit beigerechnet werden dürften; Blättchen von Biotit wurden einmal als Einschluss beobachtet.

Aller Wahrscheinlichkeit nach gehört der Allanit zu den älteren Ausscheidungen des Magmas. Einige Thatsachen und ganz besonders der gepresste Charakter des Granits sprechen für eine secundäre Entstehung des Epidots durch Druck, aber merkwïrdigerweise fand Prof. Williams, dass der epidot-allanitreiche Woodstockgranit der Umgebung keine Spur von Kataklasstructur zeigt.

Meinen hochverehrten Lehrern, Herrn Professor Dr. George H. Williams von der Johns Hopkins-Universität zu Baltimore und Herrn Geheimen Bergrath Prof. Dr. H. Rosenbusch in Heidelberg bin ich zu tiefgefiihltestem Danke verpflichtet.

Erst während des Druckes meiner Arbeit kam der Aufsatz von, A. Lacroix, Contributions ì l'étude des gneiss à pyroxène et des roches ì wernerite (Bull. de la soc. franç. de minér. XII, Nr. 4, Avril 1889) zu meiner Kenntnis. Darin beschreibt der Verfasser ganz die gleichen Verwachsungen von Allanit und Epidot aus dem Pyroxen-Amphibolgneiss von Finisterre (pag. 138-139, Fig. 21), ans dem Pyroxen-Werneritgneiss des niederösterreichischen Waldviertels (pag. 157, Pl. I, Fig. 5), sowie aus dem Werneritgneiss von Oedegaarden in Norwegen (pag. 210) und vergleicht diese mit dem Vorkommnis von Ilchester. Er hält den Epidot für primär in allen von ihm beschriebenen Vorkommnissen.

1) Prof. Willia ms findet solche Epidot-Allanitverwachsungen in dem naheliegenden Granit ron Ellicot City und Woodstock, aber nicht im weiter nach Süden liegenden Guilfordgranit. 